\title{
COMPARAÇÃO ELETROMIOGRÁFICA DOS MULTÍFIDOS NO AGACHAMENTO LIVRE E ELEVAÇÃO PÉLVICA
}

\author{
ELECTROMYOGRAPHIC COMPARASION OF MULTIFIDUS IN FREE \\ SQUAT AND HIP THRUST EXERCISE
}

\section{COMPARACIÓN ELECTROMYOGRÁFICA DE MULTIFIDES EN SENTADILLA LIBRE Y ELEVACIÓN PÉLVICA}

\author{
Eduardo Borges \\ https://orcid.org/0000-0002-7815-1365 \\ http://lattes.cnpq.br/9289971136357239 \\ Faculdade de Educação Física de Sorocaba (Sorocaba, SP - Brasil) \\ eduardo.borges2010@uol.com.br \\ Fábio Gianolla \\ https://orcid.org/0000-0003-1163-6533 (1D \\ http://lattes.cnpq.br/4724784551282078 \\ Faculdade de Educação Física de Sorocaba (Sorocaba, SP - Brasil) \\ fgianoll@gmail.com \\ Júlio Cerca Serrão \\ https://orcid.org/0000-0002-3646-3387 (iD \\ http://lattes.cnpq.br/9124685212860479 \\ Universidade de São Paulo (São Paulo, SP - Brasil) \\ jcserrao@usp.br
}

\begin{abstract}
Resumo
No melhor de nosso conhecimento, nenhum estudo da literatura comparou a ativação eletromiográfica bilateral dos músculos multífidos e longuíssimo dorsal entre o agachamento e elevação pélvica. O objetivo do presente estudo foi comparar a ativação eletromiográfica por mensuração bilateral dos músculos multífidos e longuíssimo dorsal entre os exercícios de agachamento livre e elevação pélvica em 9 voluntários, competidores universitários de fisiculturismo que estavam utilizando ambos os exercícios na rotina de treinamento. Os resultados mostraram que ambos os músculos apresentaram maior atividade eletromiográfica na fase ascendente. Com relação ao efeito do exercício, os multífidos apresentaram maior ativação na elevação pélvica $(80,9 \pm 21,4 \times 58,5 \pm 17,7$ para o pico, $p<0,05$ e $50,1 \pm 17,6 \times 34,5 \pm 12,1$ para a média, $p<0,05$ ). Não houve diferença entre os exercícios para o longuíssimo. Concluímos que os músculos multífidos são mais solicitados no exercício de elevação pélvica e ambos os exercícios apresentam maior ativação na fase ascendente.
\end{abstract}

Palavras-chave: Elevação Pélvica; Agachamento Livre; Eletromiografia; Treinamento Resistido.

\section{Abstract}

The best of our knowledge no study in the literature has compared the bilateral electromyographic activation of the multifidus and longissimus dorsi muscles between the squat and hip thrust exercise. The aim of the present study was to compare the electromyographic activation by bilateral measurement of the multifidus and longissimus dorsi muscles between the squat and hip thrust exercise in 9 university bodybuilding competitors who were using both exercises in their training routine. The results show that both muscles increased electromyography activity in the ascending phase. Regarding the exercise effect, the multifidus show greater activation in pelvic elevation $(80.9 \pm$ $21.4 \times 58.5 \pm 17.7$ for the peak, $p<0.05$, and $50.1 \pm 17.6 \times 34.5 \pm 12.1$ for the mean, $p<0.05$ ). There was no difference between the exercises for the longuissimus. We conclude that the multifidus muscles are more requested in the hip thrust exercise and both show greater activation in the ascending phase.

Keywords: Hip Thrust Exercise; Squat; Electromyography; Resistance Training. 


\section{Resumen}

Ningún estudio en la literatura ha comparado la activación electromiográfica bilateral de los músculos multifidus y longissimus dorsi entre la sentadilla y el levantamiento pélvico. El objetivo del presente estudio fue comparar la activación electromiográfica por medición bilateral de los músculos multifidus y longissimus dorsi entre los ejercicios de sentadilla libre y levantamiento pélvico en 9 competidores de culturismo universitarios que utilizaban ambos ejercicios en su rutina de entrenamiento. Los resultados mostraron que ambos músculos mostraron mayor actividad en la fase ascendente. En cuanto al efecto del ejercicio, el multífido mostró mayor activación en la elevación pélvica $(80,9 \pm 21,4 \times 58,5 \pm 17,7$ para el pico, $p<0,05$ y $50,1 \pm 17,6 \times 34,5 \pm 12,1$ para la media, $p<0,05)$. No hubo diferencia entre los ejercicios para el longissimus. Concluimos que los músculos multífidos son más solicitados en el ejercicio de elevación pélvica y ambos muestran mayor activación en la fase ascendente.

Palabras clave: Elevación Pélvica; Sentadilla Libre; Electromiografía; Entrenamiento de Resistencia.

\section{INTRODUÇÃO}

Os músculos multífidos são considerados por (BOGDUK, 2005) os principais responsáveis pelos movimentos intervertebrais. A massa dos multífidos na região lombar é superficial, e na região L4-L5 são os únicos responsáveis por mover e estabilizar a transição lombo sacral (BOJADSEN et al., 2000) e o controle das forças de cisalhamento que ocorrem durante a flexão do tronco é feita por elementos passivos e pelos multífidos da região lombar (RICHARDSON et al., 1999). Neste contexto, os exercícios de elevação pélvica e agachamento são exercícios utilizados em programas de reabilitação que envolvam os músculos eretores da coluna e multífidos com papel estabilizador (CHUNG; LEE; YOON, 2013; STEELE; BRUCE-LOW; SMITH, 2015). Uma recente revisão aponta que, exercícios que enfatizam a musculatura posterior do tronco apresenta melhores resultados para manejo de pessoas com dor lombar crônica (TATARYN et al., 2021).

O exercício de elevação pélvica apresenta uma elevada ativação do músculo longuíssimo dorsal (ANDERSEN et al., 2018) e, maior ativação deste músculo, quando comparada ao agachamento livre (NETO; VIEIRA; GAMA, 2019). No entanto, não foram encontrados estudos da literatura que compararam a ativação eletromiográfica bilateral dos músculos multífidos e longuíssimo dorsal entre os exercícios de agachamento livre e elevação pélvica, nem avaliaram as fases ascendente e descendente desses exercícios de forma independente.

Entender como os exercícios de agachamento e elevação pélvica ativa os músculos multífidos lombar e os eretores da coluna, seria uma ferramenta importante de tomada de decisão, para programas de treinamento em geral e de pessoas com dor lombar. No entanto, pessoas com dor lombar crônica apresentam assimetria dos multífidos (WALLWORK et al., 
2009; HUANG et al., 2014) com deficiência de ativação (HODGES, 2001; SILFIES et al., 2009), sendo assim, seria inviável caracterizar tais exercícios nessa população.

O objetivo do presente estudo foi comparar a ativação eletromiográfica por mensuração bilateral dos músculos multífidos e longuíssimo dorsal entre os exercícios de agachamento livre e elevação pélvica em indivíduos experientes em treinamento resistido.

\section{MATERIAIS E MÉTODOS}

\section{Sujeitos}

Nove voluntários com experiência nos exercícios agachamento e elevação pélvica (5 mulheres, 4 homens), competidores universitários de fisiculturismo sem histórico de lesão musculoesquelética participaram deste estudo. Os dados descritivos dos voluntários são apresentados na Tabela 1.

Todos os voluntários assinaram o termo de consentimento livre e esclarecido e o estudo foi aprovado pelo comitê de ética local (CAAE 86600618.4.0000.5500).

Tabela 1 - Características antropométricas dos voluntários apresentados em média $\pm D P$ ( \pm desvio padrão)

\begin{tabular}{cc}
\hline Variáveis & Média $\pm \mathrm{DP}$ \\
\hline Idade (anos) & $25,8 \pm 3,4$ \\
Estatura (cm) & $164,7 \pm 8,1$ \\
Massa corporal (kg) & $67 \pm 13$ \\
Gordura corporal (\%) & $10,4 \pm 6,8$ \\
\hline
\end{tabular}

Fonte: construção dos autores

\section{Abordagem experimental}

Estudo com um modelo crossover no qual os voluntários realizaram os exercícios: agachamento livre e elevação pélvica para obtenção de parâmetros eletromiográficos dos músculos multífidos e longuíssimo dorsal. Os voluntários compareceram em uma única sessão ao laboratório da Faculdade de Educação Física de Sorocaba para realização dos exercícios.

No dia da coleta dos dados, inicialmente foi realizada uma avaliação antropométrica dos voluntários conforme dados apresentados na Tabela 1. Na sequência, os 
voluntários foram preparados para colocação dos eletrodos nos músculos longuíssimo dorsal e multífidos, bem como a colocação de marcadores cinemáticos.

O experimento começou com a realização de um aquecimento, utilizando, 50\% da carga de 10 repetições máxima (10RM) tanto para exercício de agachamento quanto para a elevação pélvica, conforme randomização e balanceamento previamente realizados. A carga de 10 repetições máximas (10RM) foi informada pelos voluntários que, já estavam praticando os dois exercícios em preparação para uma competição de fisiculturismo universitário e, haviam ajustado as cargas de exercício uma semana antes do experimento.

No momento da coleta, para evitar um efeito de fadiga, os voluntários realizaram 4 repetições com a carga de $10 \mathrm{RM}$, tanto no agachamento quanto na elevação pélvica.

No final dos dois exercícios os voluntários realizaram uma contração isométrica voluntária máxima dos eretores da coluna contra uma resistência manual do avaliador.

\section{Execução do agachamento}

O próprio voluntário, posicionado em pé, retirou a barra de um suporte cavalete ( ${ }^{\circledR}$ Pórtico Fitness), ajustou a barra nas costas sobre os ombros, afastou os pés numa distância biacromial com rotação dos pés escolhida pela técnica habitual de cada voluntário. A partir dessa posição o indivíduo realizou a fase descendente do exercício até as coxas ficarem paralelas ao solo. Após isso, retornou à posição inicial até ficar totalmente em pé, caracterizando o final da fase ascendente do agachamento.

\section{Execução da elevação pélvica}

O voluntário, apoiou as escápulas em um banco de 1,50 m (®Pórtico Fitness) e, manteve os joelhos flexionados em $90^{\circ}$, mantendo a coluna completamente ereta e, estático na posição, aguardando que a barra fosse posicionada. Dois avaliadores posicionaram a barra logo abaixo das espinhas ilíacas superiores dos voluntários (Figura 1). Após a barra estar perfeitamente centralizada e estabilizada, o voluntário iniciava a fase descendente do exercício, por meio da flexão dos quadris e joelhos, flexionando ambas as articulações o máximo possível, até que os glúteos chegassem o mais próximo do solo. Após isso, retornava à posição inicial até a coluna ficar totalmente ereta, caracterizando o final da fase ascendente do exercício. 
Figura 1 - Voluntária posicionada para iniciar a fase descendente do exercício

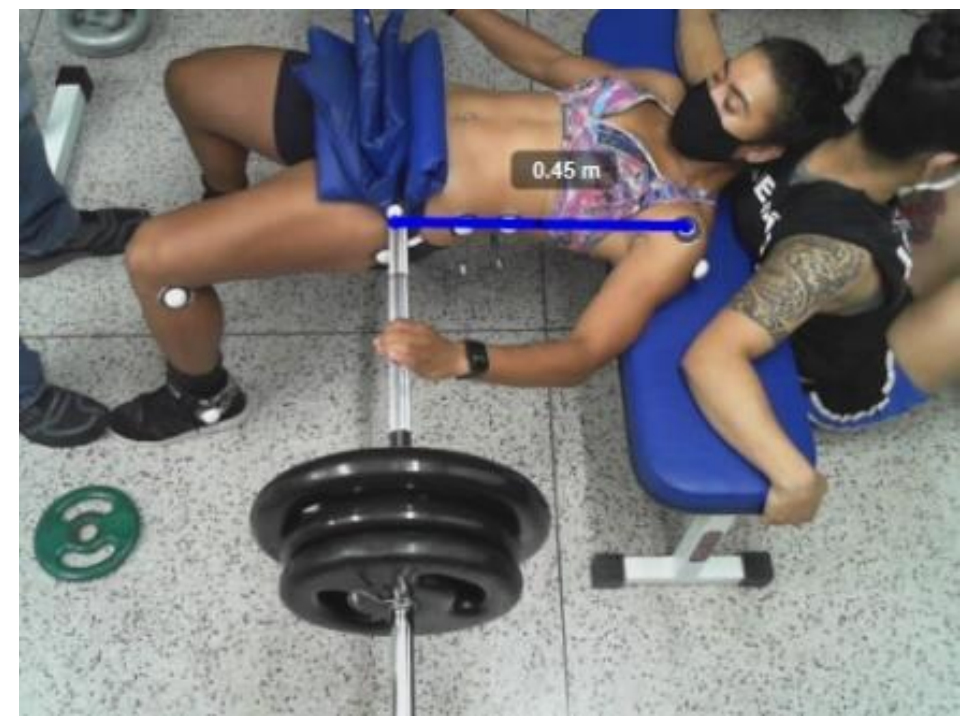

Fonte: construção dos autores

\section{Eletromiografia}

Para aquisição do sinal eletromiográfico (EMG) foi utilizado o sistema myotrace 400 (Noraxon Scottsdale, Arizona), com taxa de amostragem de 1000 Hz, conversor analógicodigital de 16 bits. Os dados foram enviados em tempo real para um computador via wireless para o software MyoResearch 3.10 master (Noraxon USA, Inc., Scottsdale, AZ).

Os eletrodos foram posicionados nos músculos longuíssimo dorsal (direito e esquerdo) e multífidos lombar (direito e esquerdo) de acordo com as recomendações da SENIAM (HERMENS et al., 1999).

O sinal EMG foi filtrado por um filtro recursivo passa banda Butterworth de quarta ordem (4thorder) com frequência de corte de 20 e $450 \mathrm{~Hz}$. Para a normalização dos dados. Foi realizado um alisamento "smoothing root mean square" (RMS) com 100ms de janelamento e a normalização dos dados foi realizada pelo maior valor RMS obtido por uma contração isométrica voluntária máxima (CIVM) de 5s. A CIVM foi realizada para cada voluntário em tentativa de estender a coluna vertebral contra uma resistência manual de um avaliador sobre as escápulas. O tratamento matemático dos dados foi realizado no software MyoResearch 3.10 master (Noraxon USA, Inc., Scottsdale, AZ).

Uma análise cinemática bidimensional foi realizada para separar as fases ascendente e descendente dos exercícios. Foi utilizado uma câmera Logitech brio ( ${ }^{\circledR}$ Logitech) com taxa de amostragem de $90 \mathrm{~Hz}$. Marcadores cinemáticos foram posicionados no lado 
esquerdo do maléolo lateral, centro articular do joelho, trocanter maior do fêmur, espinha ilíaca anterior superior, crista ilíaca e acrômio. Os dados gravados e sincronizados em tempo real com a eletromiografia por meio do software MyoResearch 3.10 master (Noraxon USA, Inc., Scottsdale, AZ).

\section{Análise estatística}

A normalidade e esfericidade dos dados foram verificadas pelos testes de Kolmogorov-Smirnov e Mauchly respectivamente. Para comparar a atividade dos músculos analisados entre os diferentes exercícios, nas fases ascendente e descendente foi utilizada a análise de variância com dois fatores para medidas repetidas, sendo os fatores o exercício (agachamento x elevação pélvica) e as fases (ascendente e descendente). Quando necessário foi utilizado o post-hoc SNK. O nível de significância adotado foi de 5\% e todas as análises foram feitas no software SigmaStat 3.5.

\section{RESULTADOS}

Os resultados das médias e pico do sinal EMG dos músculos analisados nos exercícios de agachamento livre e elevação pélvica, separados por fase descendente e ascendente são apresentados na tabela 2.

Não foi encontrada interação entre os fatores analisados (exercício e fase) para nenhuma das variáveis. Para o efeito principal da fase do exercício, ambos os músculos apresentaram maior atividade na fase ascendente (LM: $42,9 \pm 12.5 \times 35,8 \pm 10.8 ;$ LP: $69,4 \pm 16.2 \times$ 59,8 \pm 16 ; MM: $48,4 \pm 16.6 \times 36,2 \pm 15.2$; e MP: $77,1 \pm 21.7 \times 62,3 \pm 21.2$ para as fases ascendente e descendente respectivamente, $p<0,05)$. Com relação aos exercícios, os multífidos apresentaram maior ativação na elevação pélvica comparada ao agachamento $(80,9 \pm 21.4 \mathrm{x}$ $58,5 \pm 17.7$ para o pico, $p<0,05$, e 50,1 $\pm 17.6 \times 34.5 \pm 12.1$ para a média, $p<0,05$ ). Não houve diferença entre os exercícios para o longuíssimo. 
Tabela 2 - \% CIVM de ativação do sinal EMG de músculos lombares no agachamento e elevação pélvica. Dados apresentados em média e desvio padrão

\begin{tabular}{lccccccc}
\hline \multirow{2}{*}{ Músculo } & \multicolumn{2}{c}{ AGACHAMENTO } & \multicolumn{2}{c}{ ELEVAÇÃO PÉLVICA } & \multicolumn{3}{c}{ P VALOR } \\
\cline { 2 - 7 } LM & Descendente & Ascendente & Descendente & Ascendente & Exercício & Fase & Interação \\
\cline { 2 - 7 } & $34,6 \pm 7,9$ & $39,8 \pm 10,4$ & $37 \pm 13,4$ & $46,1 \pm 14,1$ & 0,271 & 0,007 & 0,055 \\
LP & $62,6 \pm 14,9$ & $72 \pm 17,7$ & $56,9 \pm 17,4$ & $66,8 \pm 15,2$ & 0,233 & 0,011 & 0,867 \\
MM & $29,6 \pm 11,1$ & $39,3 \pm 11,5$ & $42,8 \pm 16,4$ & $57,5 \pm 16,3$ & 0,025 & 0,001 & 0,065 \\
MP & $50,3 \pm 14,4$ & $66,8 \pm 17,5$ & $74,4 \pm 20,4$ & $87,5 \pm 21,3$ & 0,028 & 0,001 & 0,430 \\
\hline
\end{tabular}

Legendas: \%CIVM = Percentual da ativação eletromiográfica normalizada pela contração isométrica voluntária máxima; LM = média de ativação para o músculo longuíssimo dorsal; LP = pico de ativação para o músculo longuíssimo dorsal; $\mathrm{MM}=$ média de ativação para o músculo multífidos; MP = pico de ativação para o músculo multífido. Diferença estatística significante observados nos valores de $p<0,05$

Fonte: construção dos autores

\section{DISCUSSÃo}

O objetivo do presente estudo foi comparar a ativação eletromiográfica por mensuração bilateral dos músculos multífidos e longuíssimo dorsal entre os exercícios de agachamento livre e elevação pélvica em indivíduos experientes em treinamento resistido.

Os resultados encontrados no presente estudo, não mostraram interação significante entre os fatores analisados (exercício e fase). Assim, prosseguimos para a análise de efeito principal que, permitiu encontrar diferença significantemente maior de ativação na fase ascendente de ambos os exercícios, bem como maior ativação dos músculos multífidos na elevação pélvica.

No melhor do nosso conhecimento, este é o primeiro estudo a demonstrar uma maior ativação de músculos eretores e estabilizadores da coluna na fase ascendente dos dois exercícios. É o primeiro estudo a avaliar a ativação dos multífidos na comparação dos exercícios de agachamento livre e elevação pélvica, bem como, o primeiro a separar as fases ascendente e descendente e analisar tanto a média quanto o pico de ativação. Essas análises de forma separada permitem uma melhor compreensão dos exercícios a respeito da magnitude da ativação muscular, permitindo que os profissionais envolvidos na prescrição desses exercícios possam selecionar o exercício mais adequado a sua necessidade. Neto, Vieira e Gama (2019) não encontraram trabalhos que tenham realizado tal análise. 
Uma inspeção visual dos nossos dados, mas que não foi analisada estatisticamente, sugere um comportamento da ativação dos músculos longuíssimo dorsal e multífidos como típicos de uma contração concêntrica e excêntrica, respectivamente para as fases ascendente e descendente dos exercícios. Fato, que coincidiu com uma menor ativação na fase descendente. Tal achado, pode ser importante para a prescrição do exercício, principalmente na reabilitação. Pois, provavelmente os elementos passivos da coluna vertebral estão em maior exigência na fase descendente dos exercícios.

Andersen e colaboradores (2018) avaliaram os eretores da coluna no exercício de elevação pélvica, mas não avaliaram os multífidos e analisaram apenas a fase ascendente do movimento. Sendo que, os autores citados, encontraram um pico de ativação de aproximadamente $85 \%$ da CIVM para o músculo longuíssimo dorsal. No presente estudo, encontramos para o longuíssimo dorsal um pico de 66,8\% da CIVM na fase ascendente do movimento. Pressupomos que, Andersen e colaboradores (2018) encontraram um maior pico de ativação do longuíssimo dorsal porque os voluntários realizaram a elevação pélvica com carga para 1RM. Nós, por além de avaliar a ativação de forma bilateral, utilizamos carga equivalente a 10RM, por ser mais próximo da realidade utilizada pelos treinadores.

A maior ativação dos músculos multífidos na elevação pélvica comparada ao agachamento, tanto no pico quanto na média da ativação, provavelmente ocorre por geração de torques diferentes entre os exercícios, necessitando de mais investigações. A maior ativação dos multífidos na elevação pélvica, que foi encontrado no presente estudo, é um fato importante para treinadores que pretendam prescrever um exercício de maior solicitação para esses músculos.

\section{CONCLUSÕES}

Os músculos eretores da coluna (longuíssimo dorsal) no nível da vértebra lombar 1 (L1) não apresenta diferença significante de ativação na comparação entre os exercícios de agachamento livre e elevação pélvica. Os músculos multífidos são mais solicitados na elevação pélvica do que no agachamento livre, sendo 38\% e 45\% maior, respectivamente para pico e média de ativação. Tanto o agachamento livre quanto a elevação pélvica apresentam maior ativação na fase ascendente dos exercícios. 
Uma possível aplicação prática destas conclusões, seria realizar uma progressão do treinamento dos exercícios de agachamento e elevação pélvica, a partir da solicitação dos músculos multífidos encontrada no presente estudo. Assim, pessoas fragilizadas poderiam começar seu programa de treinamento com o exercício de agachamento livre, progredindo posteriormente para o exercício de elevação pélvica.

\section{REFERÊNCIAS BIBLIOGRÁFICAS}

ANDERSEN, Vidar e colaboradores. Electromyographic comparison of barbell deadlift, hex bar deadlift, and hip thrust exercises: a cross-over study. Journal of strength and conditioning research, v. 32, n. 3, p. 287-593, 2018.

BOGDUK, Nikolai. Clinical anatomy of the lumbar spine \& sacrum. 4th ed. London, England: Elsevier Churchill Livingstone, 2005.

BOJADSEN, Thais W. A. e colaboradores. Comparative study of $\mathrm{mm}$. multifidi in lumbar and thoracic spine. Journal of electromyography and kinesiology, v. 10, n. 3, p. 143-149, 2000.

CHUNG, Sin Ho; LEE, Ju Sang; YOON, Jang Soon. Effects of stabilization exercise using a ball on mutifidus cross-sectional area in patients with chronic low back pain. Journal of sports science and medicine, v. 12, n. 3, p. 533-541, 2013.

HERMENS, Hermie J. e colaboradores. SENIAM Project. 1999. Disponível em: <http://www.seniam.org/>. Acesso em: 30 jun. 2021.

HODGES, Paul W. Changes in motor planning of feedforward postural responses of the trunk muscles in low back pain. Experimental brain research, v. 141, n. 2, p. 261-266, 2001.

HUANG, Qiuchen e colaboradores. The evaluation of chronic low back pain by determining the ratio of the lumbar multifidus muscle cross-sectional areas of the unaffected and affected sides. Journal of physical therapy science, v. 26, n. 10, p. 1613-1614, 2014.

NETO, Walter Krause; VIEIRA, Thais Lima; GAMA, Eliane Florêncio. Barbell hip thrust, muscular activation and performance: a systematic review. Journal of sports science and medicine, $\mathrm{v}$. 18, n. 2, p. 198-206, 2019.

RICHARDSON, Carolyn e colaboradores. Therapeutic exercise for spinal segmental stabilisation in low back pain. London, England: Churchill Livingstone, 1999.

SILFIES, Sheri P. e colaboradores. Differences in feedforward trunk muscle activity in subgroups of patients with mechanical low back pain. Archives of physical medicine and rehabilitation, v. 90, n. 7, p. 1159-1169, 2009.

STEELE, James; BRUCE-LOW, Stewart; SMITH, Dave. A review of the specificity of exercises 
designed for conditioning the lumbar extensors. British Journal of sports medicine, v. 49, n. 5, p. 291-297, 2015.

TATARYN, Nicholas e colaboradores. Posterior-chain resistance training compared to general exercise and walking programmes for the treatment of chronic low back pain in the general population: a systematic review and meta-analysis. Sports medicine - open, v. 7, n. 1, 2021.

WALLWORK, Tracy L. e colaboradores. The effect of chronic low back pain on size and contraction of the lumbar multifidus muscle. Manual therapy, v. 14, n. 5, p. 496-500, 2009.

\section{Dados do primeiro autor:}

Email: eduardo.borges2010@uol.com.br

Endereço: Rua Renato Swensson, 93, Parque Três Meninos, Sorocaba, SP, CEP 18016-220, Brasil.

Recebido em: 30/06/2021

Aprovado em: 24/07/2021

\section{Como citar este artigo:}

BORGES, Eduardo; GIANOLLA, Fábio; SERRÃO, Júlio Cerca. Comparação eletromiográfica dos multífidos no agachamento livre e elevação pélvica. Corpoconsciência, v. 25, n. 2, p. 186-195, mai./ ago., 2021. 